\title{
A machine-learning approach to predict postprandial hypoglycemia
}

\author{
Wonju Seo ${ }^{1 \dagger}$, You-Bin Lee ${ }^{2 \dagger}$, Seunghyun Lee ${ }^{1}$, Sang-Man Jin ${ }^{2 *}$ and Sung-Min Park ${ }^{*}$ (D)
}

\begin{abstract}
Background: For an effective artificial pancreas (AP) system and an improved therapeutic intervention with continuous glucose monitoring (CGM), predicting the occurrence of hypoglycemia accurately is very important. While there have been many studies reporting successful algorithms for predicting nocturnal hypoglycemia, predicting postprandial hypoglycemia still remains a challenge due to extreme glucose fluctuations that occur around mealtimes. The goal of this study is to evaluate the feasibility of easy-to-use, computationally efficient machine-learning algorithm to predict postprandial hypoglycemia with a unique feature set.

Methods: We use retrospective CGM datasets of 104 people who had experienced at least one hypoglycemia alert value during a three-day CGM session. The algorithms were developed based on four machine learning models with a unique data-driven feature set: a random forest (RF), a support vector machine using a linear function or a radial basis function, a K-nearest neighbor, and a logistic regression. With 5-fold cross-subject validation, the average performance of each model was calculated to compare and contrast their individual performance. The area under a receiver operating characteristic curve (AUC) and the F1 score were used as the main criterion for evaluating the performance.

Results: In predicting a hypoglycemia alert value with a 30-min prediction horizon, the RF model showed the best performance with the average AUC of 0.966 , the average sensitivity of $89.6 \%$, the average specificity of $91.3 \%$, and the average F1 score of 0.543 . In addition, the RF showed the better predictive performance for postprandial hypoglycemic events than other models.

Conclusion: In conclusion, we showed that machine-learning algorithms have potential in predicting postprandial hypoglycemia, and the RF model could be a better candidate for the further development of postprandial hypoglycemia prediction algorithm to advance the CGM technology and the AP technology further.
\end{abstract}

Keywords: Hypoglycemia, Risk prediction, Machine-learning approach, Diabetes

\section{Background}

Intensive insulin treatment is a standard of care for tight glycemic control in people with diabetes, to prevent or delay long-term complications of diabetes mellitus [1-3] However, insulin therapy may cause life threatening hypoglycemia and thus achieving and maintaining near normoglycemia is largely limited by this risk factor [4], which persists despite advances in treatment technique [2-6]. Recently, artificial pancreas (AP) systems

\footnotetext{
*Correspondence: smj0919@skku.edu; sungminpark@postech.ac.kr

${ }^{\dagger}$ Wonju Seo and You-Bin Lee contributed equally to this work.

'Department of Creative IT engineering, POSTECH, 77, Cheongam-Ro,

Nam-Gu, 37673 Pohang, Republic of Korea

${ }^{2}$ Division of Endocrinology and Metabolism, Department of Medicine,

Samsung Medical Center, Sungkyunkwan University School of Medicine, 81, Irwon-ro, 06351 Seoul, Republic of Korea
}

(C) The Author(s). 2019 Open Access This article is distributed under the terms of the Creative Commons Attribution 4.0 International License (http://creativecommons.org/licenses/by/4.0/), which permits unrestricted use, distribution, and reproduction in any medium, provided you give appropriate credit to the original author(s) and the source, provide a link to the Creative Commons license, and indicate if changes were made. The Creative Commons Public Domain Dedication waiver

(http://creativecommons.org/publicdomain/zero/1.0/) applies to the data made available in this article, unless otherwise stated. to reduce the frequency of hypoglycemic episodes, even in the presence of intensive insulin treatment, and are among the greatest advances in diabetes care in recent development $[7,8]$.

In the clinically approved hybrid AP system, mealtime insulin dosing depends on the carbohydrate counting by patients. This process requires extensive patient education and is a complex task to most patients, which can lead to entering inaccurate information and consequently miscalculation of insulin dosage [9]. For this reason, the single-hormone AP system delivers a reduced bolus insulin dose at mealtime, to avoid risk of postprandial hypoglycemia. The systems then reduce the postmeal hyperglycemia by increasing the infusion rate of 
basal insulin. This conservative dosing of mealtime bolus insulin explains in part why only modest daytime benefit has been achieved by the single-hormone AP system, in spite of impressive nocturnal glucose control. The recently proposed bi-hormonal AP system [6] controls both insulin and glucagon pump to reduce hypoglycemia while maintaining intensive insulin treatment, and thus does not require carbohydrate counting by patients. However, the use of an additional glucagon pump increases the system cost and complexity. Furthermore, the use of these systems can cause nausea, and raises long-term concerns about safety [10, 11]. It has been reported that single- and bi-hormonal artificial pancreas systems indeed control nocturnal glucose with equal effectiveness [12]. Despite the existence of algorithms that predict nocturnal hypoglycemia (for both single- and bi-hormonal AP systems) with high accuracy, the prediction of postprandial hypoglycemia is still a challenge because of extreme glucose fluctuations around mealtimes. Current rapid-acting insulin [13] analogues are still not equivalent to physiologic biphasic insulin secretion that precisely regulates the rapid changes in glucose intake, glucagon secretion, endogenous glucose production, and utilization of glucose around mealtimes[14]. This highlights the importance of developing an accurate and easy to use algorithm to predict postprandial hypoglycemia.

As a method for predicting a glucose level or the occurrence of the hypoglycemia, a physiological prediction method, a data-driven method, and a hybrid method were proposed $[15,16]$. Among them, many data-driven models relying on continuous glucose monitoring (CGM) data and additional inputs such as diet, exercise, insulin injection dosage, and others have been proposed [15]. Firstly, there have been several studies to develop time series models [17-19] that can predict a glucose level. Sparacino et al. [17] proposed a first-order autoregressive (AR) and a first-order polynomial model to predict a glucose level with 30 -min or 45 -min prediction horizons. The proposed forgetting factor allowed the models to be more sensitive to the recent CGM data points, and thus the models were able to reflect rapid glucose changes around mealtimes. However, the models needed to tune their parameters at every sampling and the predicted glucose fluctuated significantly as the forgetting factor got smaller. An autoregressive integrated moving-average (ARIMA) model with an adaptive algorithm was recently proposed [18]. When the proposed model predicted hypoglycemia with the 30min prediction horizon, time of detection was earlier and false alarm rate was lower than other models such as an adaptive univariate model and a general ARIMA model. However, this model requires large computations and is difficult to be implemented in real time.
Another time series model with exogenous inputs such as meal information, insulin on board, and physical activity was recently proposed [19]. Although this model showed impressive performance for an early prediction of hypoglycemia, requirements for manual inputs makes this solution cumbrous, difficult to use and prone for errors [16].

Machine learning has made a great progress in medical data science and has been applied to a glucose level or hypoglycemia prediction as well [15, 16, 20-28]. The artificial neural network (ANN) proposed in [20] used five consecutive CGM data points and measurement time. Although ANN showed better predictive performance than time series model (e.g., AR model [17]), it could not detect sudden changes in a glucose level due to meals or insulin injections. However, this limitation could be overcome by inserting additional information such as meals [21-23] and insulin doses [23], but the cost of simplicity. A feed-forward neural network model has been presented in [24], but training this model also required burdensome inputs such as insulin dosage, nutritional intake, lifestyle, and emotional factor other than consecutive CGM data points. In addition to the neural network approach, there have been numerous efforts to predict hypoglycemia with an ensemble method [25], a decision-tree method $[26,27]$, and machine learning models using selfmonitoring blood glucose (BG) data [28]. However, most of the related works required more than one form of manual inputs for more accurate prediction of hypoglycemia and did not focus on postprandial hypoglycemia except for our preliminary study [27].

The goal of this study is to develop an easy-to-use, computationally efficient machine-learning algorithm to predict postprandial hypoglycemia using unique datadriven features derived from the data. The glucose dynamics in daytime and nighttime are quite different due to meals and activities, and having a dedicated algorithm for the postprandial hypoglycemia would improve the AP's insulin dosing during the daytime benefitting the patient significantly. In addition, computationally efficient algorithm, which does not require any parameter tuning on new dataset, with the reduced number of manual inputs will help the AP system to be more usable, compact, and clinically feasible. We validated postprandial hypoglycemia prediction with $30 \mathrm{~min}$ of the prediction horizon using retrospective CGM dataset of 104 patients described in the data "Data acquisition" section. As detailed in the "Methods" section, we used commonly used machine learning models to compare and contrast their individual performance, and finally determine the best candidate for the further development of the postprandial hypoglycemia prediction algorithm for the future AP system. In the next few subsections we present our work in greater detail. 


\section{Methods}

\section{Data acquisition}

We reviewed the medical records of all 411 patients who underwent CGM at Samsung Medical Center (Seoul, Republic of Korea) between 2013 and 2015, with approval from the Institutional Review Board (IRB) of the Samsung Medical Center (IRB File number; SMC 201605-058-001). All medical procedures were performed in accordance with relevant guidelines and regulations. The informed consent requirement for this study was waived by the board because the researcher only accessed the database for analysis purposes, and all patient data were de-identified. From these records, we extracted 113 three-day CGM datasets from 110 Korean adults (aged $\geq 18$ y) that met two inclusion criteria: 1) the CGM data contained at least one BG level $\leq 3.9 \mathrm{mmol} / \mathrm{L}$ $(70 \mathrm{mg} / \mathrm{dL})$; and 2) the primary purpose of performing CGM included evaluation of hypoglycemia. Of the 110 patients who met inclusion criteria, six were excluded: two patients had alimentary hypoglycemia after total gastrectomy, two patients took acetaminophen during the CGM, one patient had gestational diabetes and was not taking any anti-diabetic medications or insulin $(\mathrm{n}=1)$, and one patient had missing data. Of the remaining 104 patients (Table 1), 52 (aged 18-74 years) had type-1 diabetes mellitus and 52 (aged 32-80 years) had type- 2 diabetes mellitus. During the study, none of the patients was under steroid therapy with doses higher than a prednisolone equivalent of $7.5 \mathrm{mg}$ per day, and none was under cancer treatment such as chemotheraphy or radiotheraphy. One patient was undergoing treatment for diabetic foot infection. Three of the participants contributed two separate three-day CGM datasets separated by 9 to 22 months, so a total of 107 three-day CGM datasets were used.

The Medtronic's CGMS Gold ${ }^{T M}$ (Medtronic MiniMed, Northridge, CA, USA) was used to collect continuous glucose data for the durations of 72-96 h with subjects blinded to the data. The CGMS Gold report indicated mean absolute relative difference (MARD) values of 11 to $14 \%$, and a continuous BG error grid plot analysis showed percentage paired values in zone A (optimal) or zone B (acceptable from a clinical/diabetological perspective) to be $98 \%[29,30]$. BG trends and patterns were identified by retrospective analysis of CGM data. To calibrate the CGM sensors, finger-stick BG levels were measured more than three times per day in all enrolled patients with each measurement made right before each meal. Therefore, the time points at which the BG measurements were recorded for calibration purpose were regarded as the mealtime for this study. The BG measurements within the nocturnal interval (11:00 PM - 7:00 AM) [31] were excluded for the purpose of this study. In addition, if the time interval between two successive BG measurements was less than
$2 \mathrm{~h}$, only the last measurement was considered as a meal announcement.

We used Excel (Microsoft, Redmond, USA) to prepare the CGM data and Matlab (Matlab release 2016b, The MathWorks, Natick, 2016) to implement machine learning models and their analyses.

\section{Data pre-processing and feature extraction}

Each CGM time series was presented as a sequence where the $i^{\text {th }}$ CGM time series is given by:

$$
C G M_{i,:}=\left\{\left(C G M_{i, t}\right) \text { with } t=1, \ldots, N_{i}\right\}
$$

where $N_{i}$ is the length of $C G M_{i, .}$. For each time series, missing CGM data points were interpolated by the spline method [20] only if less than 3 CGM data points were missing consecutively. The missing CGM data points were reported when the device fails its calibration process [32]. The CGM measurement is taken at every $5 \mathrm{~min}$, and thus $C G M_{i, t=n}$ means that the CGM data point at $5 \times n^{\text {th }}$ minute of the $i^{\text {th }}$ CGM time series. In our study, we took CGM data points after meal announcements and each CGM data point is represented in Eq. 2.

$$
C G M_{i, j, t}=C G M_{i, \text { meal }_{i, j}+t} \text { with } t \in\{1, \ldots, W\}
$$

where meal $_{i, j}$ is the time of the $j^{\text {th }}$ meal announcement of the $i^{\text {th }}$ CGM time series, and $W$ is the postprandial period.

We first analyzed the CGM trends of all selected patients' data to identify meaningful features for postprandial hypoglycemia. A subset of patients experienced postprandial hypoglycemia if they had a small peak or no peak in CGM, probably due to the meal being small or containing only a small portion of carbohydrate (Fig. 1a and $b$ ). There was another group of patients experienced hypoglycemia when the CGM increased steeply and then dropped right after the peak; this reaction probably occurred when the patients ingested carbohydrates with high glycemic index or when the pre-meal rapid-acting insulin was injected too late (Fig. 1c). Insulin injected before a preceding meal can affect a glucose level after the meal. In other cases, a decrease in CGM, in spite of meal ingestion, may have been caused by the insulin on board and was associated with future hypoglycemic episodes (Fig. 1d).

We used above observed data points to define features for predicting hypoglycemia near mealtime. The first feature is defined as 'the rate of increase in glucose' (RIG), which is the rate of glucose increase from a meal to a peak:

$$
R I G_{i, j, t}=\frac{C G M_{i, j, p e a k_{t}}-C G M_{i, j, 0}}{T D_{\text {meal-to-peak }}}
$$

where $C G M_{i, j, p e a k_{t}}$ is the highest CGM data point between the time of the $j^{\text {th }}$ meal announcement of the $i^{\text {th }}$ CGM time series and prediction time $t, C G M_{i, j, 0}$ is a CGM data 
Table 1 Clinical characteristics of enrolled study subjects

\begin{tabular}{|c|c|c|}
\hline & $\begin{array}{l}\text { Type-1 diabetes ( } 55 \text { three-day } \\
\text { CGM datasets in } 52 \text { patients) }\end{array}$ & $\begin{array}{l}\text { Type-2 diabetes ( } 52 \text { three-day } \\
\text { CGM datasets in } 52 \text { patients) }\end{array}$ \\
\hline Age (year) & $40.0(29.0-52.0)$ & $63.5(54.3-68.0)$ \\
\hline Sex (male:female) & $21: 34^{*}$ & $21: 31$ \\
\hline Body weight (kg) & $60.48(52.35-69.41)$ & $60.75(54.60-70.37)$ \\
\hline $\mathrm{BMI}\left(\mathrm{kg} / \mathrm{m}^{2}\right)$ & $22.85 \pm 3.26$ & $24.60 \pm 2.62$ \\
\hline Duration of diabetes (years) & $11.0(6.0-18.0)$ & $19.0(13.3-25.0)$ \\
\hline $\begin{array}{l}\text { Insulin therapy (with insulin ther- } \\
\text { apy: without insulin therapy) }\end{array}$ & $55^{*}: 0$ & $43: 9$ \\
\hline $\begin{array}{l}\text { Insulin regimen basal:intermediate- } \\
\text { acting: premix:MDI:CSII }\end{array}$ & $3: 1: 6: 44: 1$ & 20:3:11:9:0 \\
\hline Daily insulin dose (IU/day) & $42.3 \pm 17.7$ & $28.6 \pm 18.1$ \\
\hline $\begin{array}{l}\text { Daily insulin dose per body weight } \\
\text { (IU/day/kg) }\end{array}$ & $0.68(0.53-0.82)$ & $0.50(0.30-0.60)$ \\
\hline $\mathrm{eGFR}\left(\mathrm{ml} / \mathrm{min} / 1.73 \mathrm{~m}^{2}\right)$ & 83.05 (71.98-96.95) & $70.40(51.30-82.50)$ \\
\hline End stage renal disease [n (\%)] & $4(7.3)$ & $2(3.8)$ \\
\hline Liber cirrhosis [n (\%)] & $2(3.6)$ & $0(0.0)$ \\
\hline $\begin{array}{l}\text { Heart failure with reduced ejection } \\
\text { fraction }[\mathrm{n}(\%)]\end{array}$ & $0(0.0)$ & $1(1.9)$ \\
\hline Pancreatic resection [n (\%)] & $2(3.6)+$ & $0(0.0)$ \\
\hline Acute infection [n (\%)] & $0(0.0)$ & $1(1.9)$ \\
\hline Pregnancy [n (\%)] & $1(1.8)$ & $0(0.0)$ \\
\hline Hemoglobin A1C (\%) & $7.94 \pm 1.13$ & $8.31 \pm 1.32$ \\
\hline C-peptide (ng/mL) & $0.02(0.02-0.15)$ & $1.46(0.80-2.44)$ \\
\hline
\end{tabular}

Continuous variables with normal distributions are expressed as mean \pm standard deviation, whereas continuous variables with non-normal distributions were expressed as median (interquartile range)

*Three female patients on insulin therapy were included twice because they participated twice

† One of these two patients underwent total pancreatectomy; the other went through Whipple's operation. Abbreviations: CGM, continuous glucose monitoring; BMI, body mass index; MDI, multiple daily injections; CSII, continuous subcutaneous insulin infusion; eGFR, estimated glomerular filtration rate

point at the $j^{\text {th }}$ meal announcement, and $T D_{\text {meal-to-peak }}$ is time difference between the meal announcement to the peak. The RIG is updated until the peak CGM data point is found after the meal announcement. If there is no peak CGM data point, the RIG is set to 0 . According to the definition of the RIG, zero implies that there is no increase in glucose after the meal.

Since the change in CGM data points is large before hypgolycemia occurs (Fig. 1), we defined the second feature glucose rate of change (GRC) as:

$$
G R C_{i, j, t}=\frac{C G M_{i, j, t}-C G M_{i, j, t-1}}{5}
$$

where $C G M_{i, j, t}$ is a CGM data point at the time of prediction from the $j^{\text {th }}$ meal announcement of the $i^{\text {th }}$ CGM time series, and $C G M_{i, j, t-1}$ is the CGM data point immediately prior to the time of prediction. Since the GRC calculates the near-instantaneous changes in CGM data points around the time of prediction, it can be used to predict hypoglycemia [26,33]. The third feature is defined to be the CGM data point at the time of prediction $\left(C G M_{i, j, t}\right)$ from the $j^{\text {th }}$ meal announcement of the $i^{\text {th }}$ CGM time series. To define labels, we took into account the presence of a hypoglycemia alert value [34, 35] at the 30-min prediction horizon (i.e., $C G M_{i, j, t+6}$ ). If $C G M_{i, j, t+6}<3.9$ $\mathrm{mmol} / \mathrm{L}(70 \mathrm{mg} / \mathrm{dL})$, we set Label $_{i, j, t}=1$. Otherwise, we set Label $_{i, j, t}=0$ (Fig. 2).

We obtained all available CGM data points between $5 \mathrm{~min}$ and $3.5 \mathrm{~h}$ post mealtime announcements (i.e., from $C G M_{i, j, 1}$ to $C G M_{i, j, 42}$ ). The corresponding hypoglycemia alert values that occur from $35 \mathrm{~min}$ to $4 \mathrm{~h}$ after meal announcements were included (i.e., from Label $_{i, j, 1}$ to Label $_{i, j, 42}$ ). Although postprandial hypoglycemia can occur later than $4 \mathrm{~h}$ after each meal, we chose the window of $35 \mathrm{~min}$ to $4 \mathrm{~h}$ after the meal because including longer duration after the meal to this time window decreases the prediction accuracy of the algorithm. Since there are already well-established algorithms for predicting fasting or nocturnal hypoglycemia $[25,36]$, a clinical need of a dedicated algorithm for predicting postprandial hypoglycemia would be most important during the first $4 \mathrm{~h}$ after each meal, which is typically difficult to 


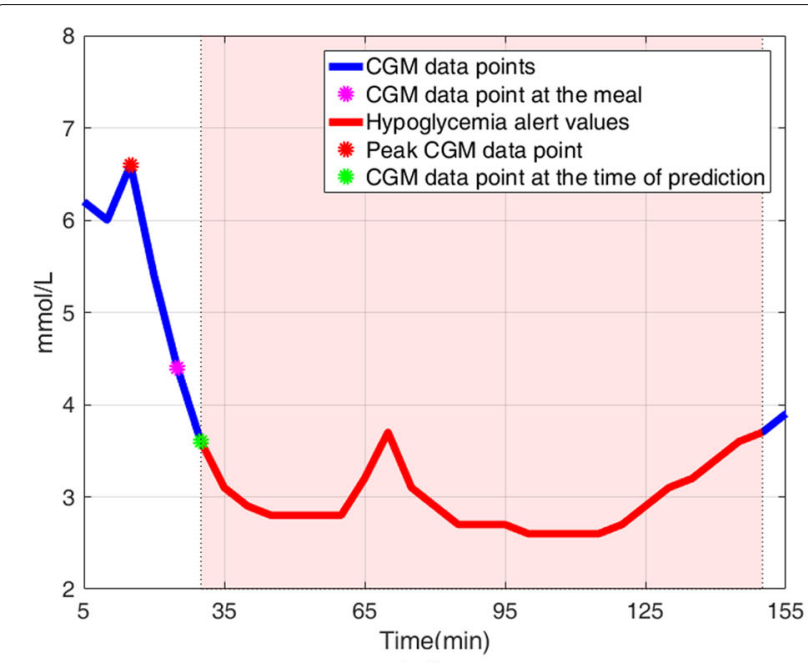

(a)

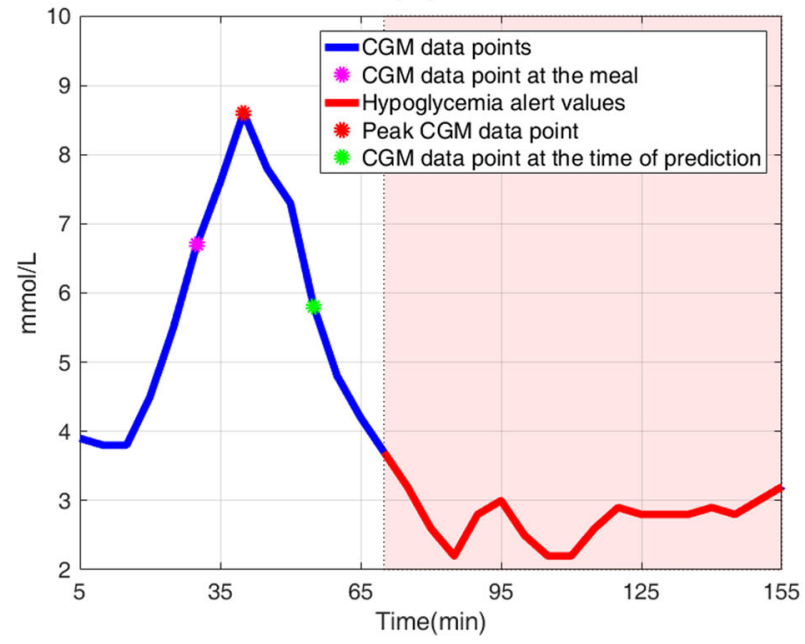

(c)

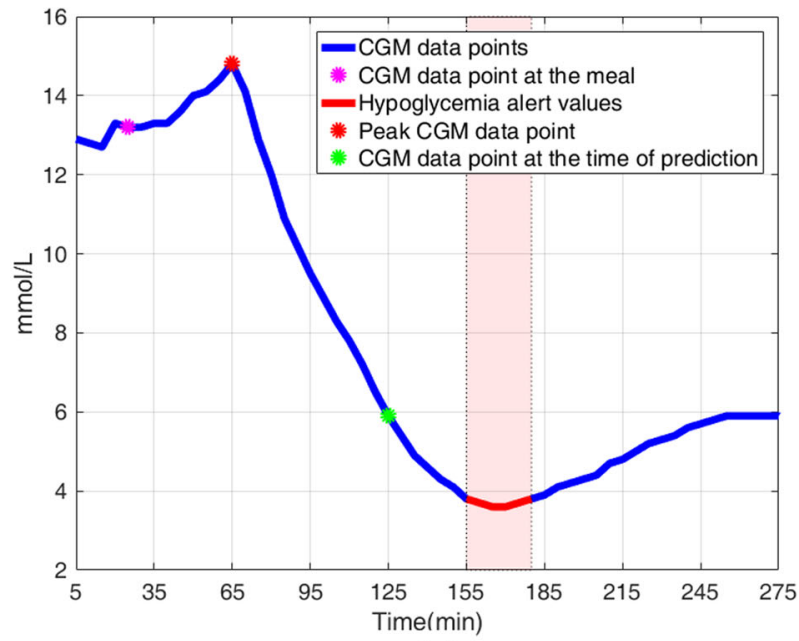

(b)

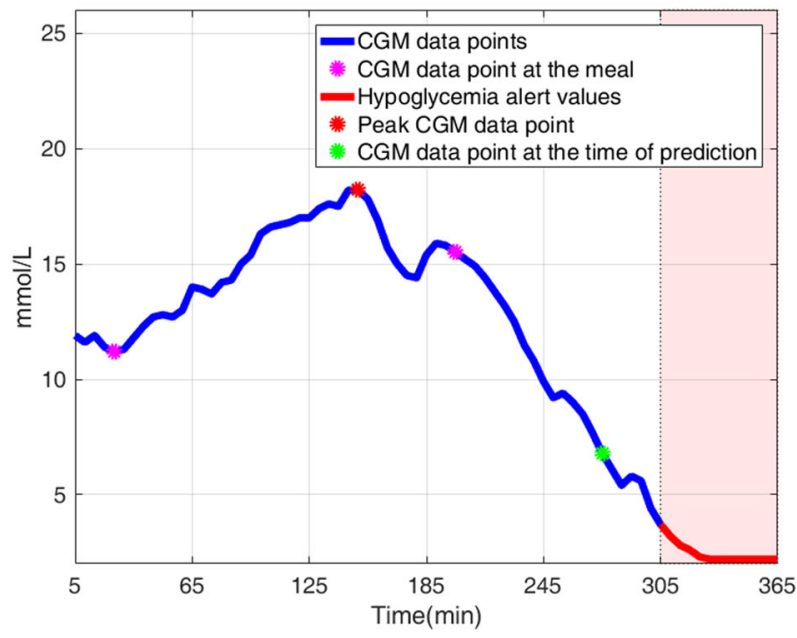

(d)

Fig. 1 Representative CGM time-series data to show different reactions of selected patients' glucose levels after meals. Blue line: CGM time-series data points; red line and transparent red box: CGM data point $<3.9 \mathrm{mmol} / \mathrm{L}(70 \mathrm{mg} / \mathrm{dL})$; magenta filled circle: CGM data point at the meal; red filled circle: peak CGM data point after the meal; green filled circle: CGM data point at the time of prediction. Clinical explanations: a No peak of CGM data point could occur because the patient ate a small amount of carbohydrates in the meal. $\mathbf{b}$ Low peak after the meal, then rapid fall in glucose could occur because patient ate a small amount of carbohydrates in the meal. c Steep peak, then rapid fall in glucose could occur when the patient ate foods rich in carbohydrate with high glycemic index or injected rapid-acting insulin later than he or she should have. $\mathbf{d}$ A rapid fall and then no peak after the meal could occur when the insulin injected before the previous meal is still active (insulin on board)

cover using the existing nocturnal hypoglycemic prediction algorithms developed in the setting of gradual changes of blood glucose levels.

The data processing and the feature extraction were performed using the following steps : First, from the $i^{t h}$ CGM time series, the $j^{t} h$ meal announcement is selected and the CGM data points from $C G M_{i, j, 1}$ to $C G M_{i, j, 42}$ were sampled. Second, from the sampled series, $C G M_{i, j, t}, R I G_{i, j, t}$, and $G R C_{i, j, t}$ features were extracted while increasing $t$ from 1 to 42. The label information is obtained from the CGM data point with the 30 -min prediction horizon (i.e., $\left.C G M_{i, j, t+6}\right)$.
The first and second steps were repeated for 107 CGM time series around mealtimes, and obtained samples :

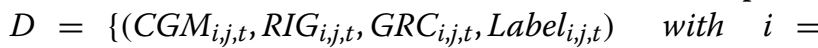
$1, \ldots, 107, j=1, \ldots, M_{i}$, and $\left.t=1, \ldots, 42\right\}$, where $M_{i}$ is the total number of meal announcements of the $i^{\text {th }}$ CGM time series. Before training our models, each feature values extracted were normalized with a MinMax Scaler.

\section{Models}

In this study, we selected four commonly used machine learning models with the unique data-driven feature set to predict the occurrence of postprandial hypoglycemia at 


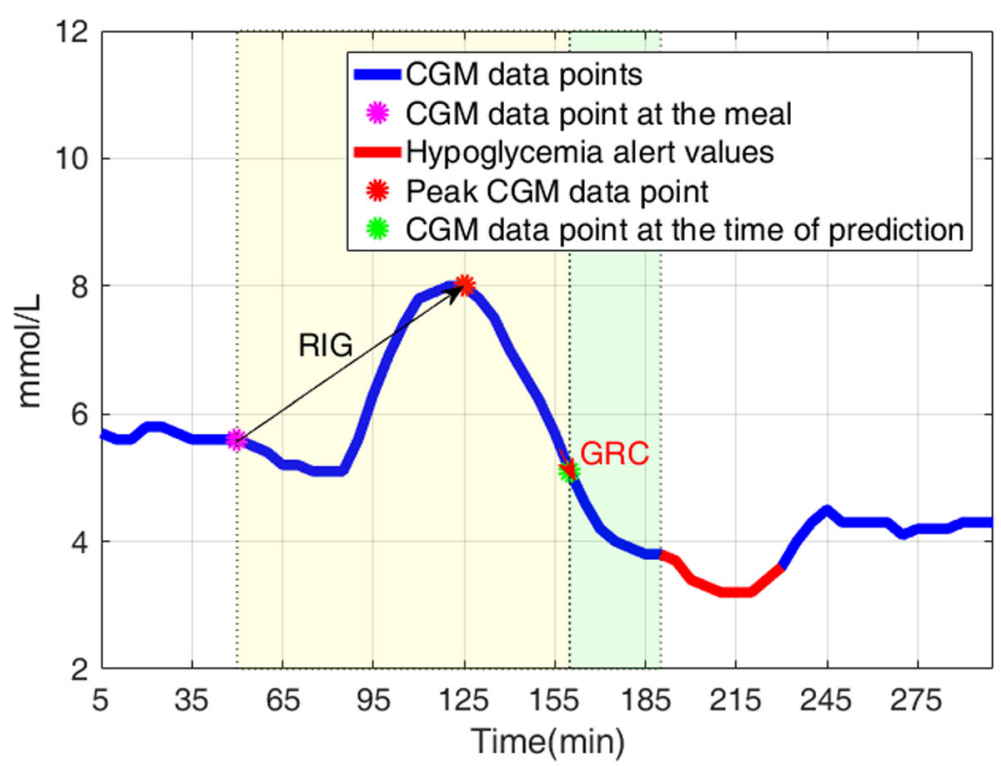

Fig. 2 The three features and the 30-min prediction horizon. Blue line: CGM time-series data points; red line: CGM data point <3.9 mmol/L (70 mg/dL); magenta filled circle: CGM data point at the meal; red filled circle: peak CGM data point after the meal; green filled circle: CGM data point at the time of prediction; black arrow: rate of increase in glucose (RIG); red arrow: glucose rate of change (GRC); transparent yellow box: observational window; transparent green box: the 30-min prediction horizon

$30 \mathrm{~min}$ from the time of prediction. Four machine learning models were selected [28, 37]: a random forest (RF), a support vector machine using a linear function (SVM$\mathrm{LN}$ ) or a radial basis function (SVM-RBF), and a K-nearest neighbor (KNN). Since there are few studies using a logistic regression (LR) $[15,16]$, we additionally considered the algorithm.

To train and evaluate each model, we used 5-fold crosssubject validation by splitting $D$ into train set $_{q}$ and test set $_{q}$ for $q^{\text {th }}$ iteration. The train set $_{q}$ and the test set $_{q}$ were represented as following:

1 train $\operatorname{set}_{q}=$ $\left\{\left(C_{i, j, t}, R I G_{i, j, t}, G R C_{i, j, t}\right.\right.$, Label $\left._{i, j, t}\right)$ with $i \in$ $\left.R_{q}, j=1, \ldots, M_{i}, t=1, \ldots, 42\right\}, R_{q}$ is the $q^{t h}$ training folds.

2 test set $_{q}=$ $\left\{\left(C G M_{i, j, t}, R I G_{i, j, t}, G R C_{i, j, t}\right.\right.$, Label $\left._{i, j, t}\right)$ with $i \in\left(R_{q}^{c} \cap\right.$ total CGM dataset), $\left.j=1, \ldots, M_{i}, t=1, \ldots, 42\right\}$.

Data imbalance should be considered before training a model with train set $q$. This imbalance results when the model is mainly trained to predict a majority class and cannot predict a minority class. It is important to predict the minority class since it is the main target in most cases, e.g., postprandial hypoglycemia. For the model to be trained, we chose a false-positive cost and a falsenegative cost so that it does not focus on the majority class based on imbalanced data handling [38]. In the training, cost of false negative was determined using Eq. 5 on train $\operatorname{set}_{q}$, cost of false-positive was set to 1, true-negative cost and true-positive cost were set to 0 .

Cost of false negative $=\frac{\sum_{\mathrm{i} \in \mathrm{R}_{\mathrm{q}}} \sum_{\mathrm{j}=1}^{\mathrm{M}_{\mathrm{i}}} \sum_{\mathrm{t}=1}^{42}\left(1-\text { Label }_{\mathrm{i}, \mathrm{j}, \mathrm{t}}\right)}{\sum_{\mathrm{i} \in \mathrm{R}_{\mathrm{q}}} \sum_{\mathrm{j}=1}^{\mathrm{M}_{\mathrm{i}}} \sum_{\mathrm{t}=1}^{42}\left(\text { Label }_{\mathrm{i}, \mathrm{j}, \mathrm{t}}\right)}$

Then, we optimized each model's hyperparameters with grid search. The best hyperparameters set that resulted in the lowest cross-validation loss, were used for training. During testing, after the $j^{\text {th }}$ meal announcement of the $i^{\text {th }}$ CGM time series, $C G M_{i, j, t}, R I G_{i, j, t}$, and $G R C_{i, j, t}$ were extracted at the time of prediction $t$. The features were normalized by the MinMax Scaler trained on a train set. The trained models predict postprandial hypoglycemia with the features. This process was repeated for all $t$ increasing from 1 to 42 . The number of cases that accurately predicted label vs. inaccurately predicted label were calculated for all meal announcements of the CGM time series in the test set.

The average performance was calculated with the 5fold cross-subject validation on 107 CGM time series. All training and evaluating process is summarized in the flowchart shown in Fig. 3.

\section{Metrics}

We used four statistical metrics for evaluating the performance of each model: the area under a curve (AUC) of a receiver operating characteristic (ROC) curve, sensitivity, specificity, and F1 score. The AUC for each model is independent of cut-off values on the ROC curve and is mainly 


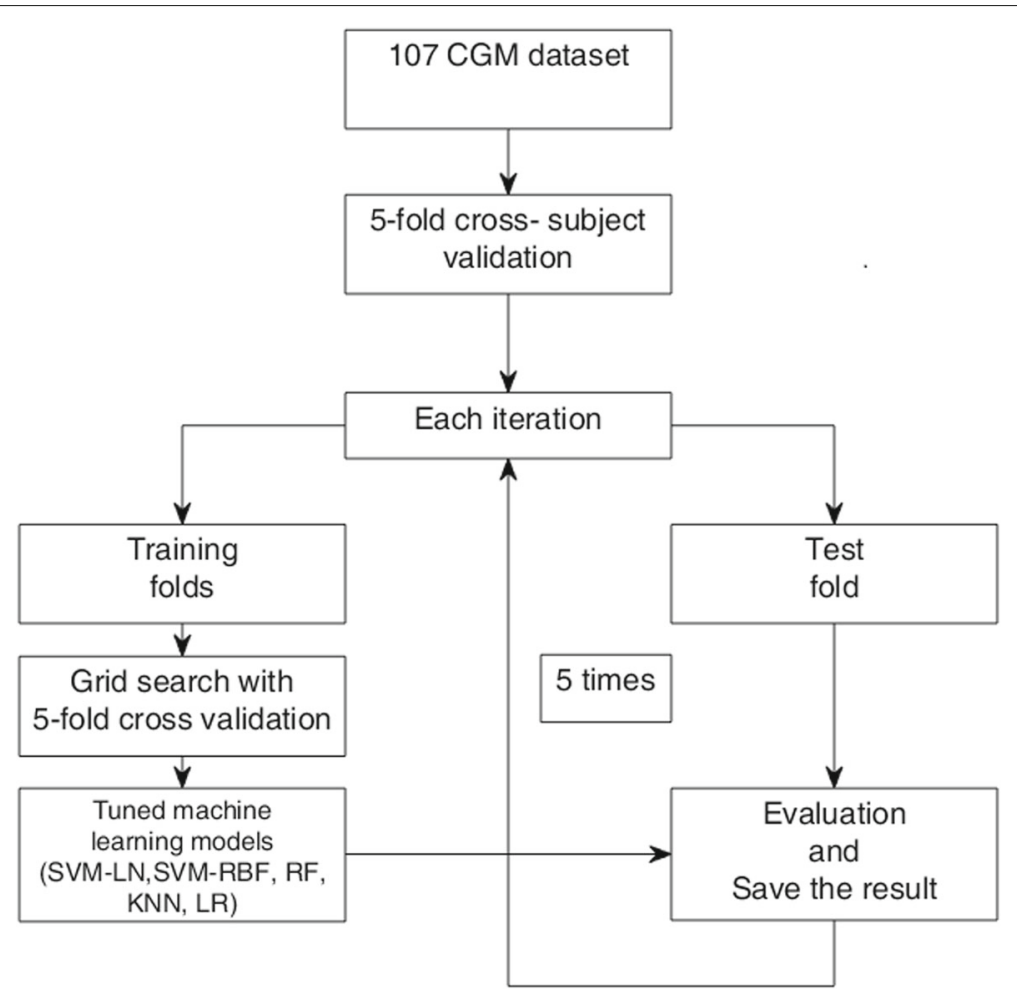

Fig. 3 Flowchart of the proposed approach including data-preprocessing, and how to train RF, SVM-LN, SVM-RBF, KNN, and LR. Since the 5-fold cross-subject validation was used, training and testing models were repeated by 5 times. For each iteration, each model's result and tuned hyper-parameters was saved

used to compare the predictive capabilities of the models, and the AUC is used as the main criterion for indicating the accuracy of the model [39]. Other metrics are defined as follows:

$$
\begin{gathered}
\text { Sensitivity }(\text { or Recall })=\frac{T P}{T P+F N} \\
\text { Specificity }=\frac{T N}{T N+F P} \\
\text { Precision }=\frac{T P}{T P+F P} \\
\text { F1 score }=2 \times \frac{\text { Precision } \times \text { Recall }}{\text { Precision }+ \text { Recall }}
\end{gathered}
$$

The true positive $(T P)$ represents the number of cases predicted correctly to have a hypoglycemia alert value (CGM data point $<3.9 \mathrm{mmol} / \mathrm{L}$ ) at $30 \mathrm{~min}$ from the time of prediction and the true negative $(T N)$ indicates the number of cases predicted correctly to not have a hypoglycemia alert value at $30 \mathrm{~min}$ from the time of prediction. The false positive $(F P)$ is the number of cases predicted falsely to have a hypoglycemia alert value at $30 \mathrm{~min}$ from the time of prediction, and false negative $(F N)$ is the number of cases predicted incorrectly to have a hypoglycemia alert at $30 \mathrm{~min}$ from the time of prediction. Sensitivity (or Recall) measures the ability of the algorithm to correctly detect hypoglycemia while specificity measures the ability of the algorithm to correctly detect the absence of it. F1 score is the harmonic mean of both metrics of recall and precision. Since there is a trade-off relationship between precision and recall, F1 score helps to consider both metrics.

To calculate the performance of predictions, the definition of a hypoglycemic event and the definition of an alarm as defined in [19] are used. The main difference between our study and prior study [19] is that we only consider hypoglycemic events in postprandial time interval (5 min to $4 \mathrm{~h}$ after a meal). A hypoglycemic event typically has more than two consecutive hypoglycemia alert values. If the time difference between the two events is more than 10 min (two-time steps), the two events are considered as different events. Otherwise, the two events are merged to form the same event. In true alarm, the trained model would predict a hypoglycemia alert value continuously (more than two-time steps) within $60 \mathrm{~min}$ before the hypoglycemic event. The detection time (DT) is the time between the first time of an alarm and the first time of a hypoglycemic event. We did not consider alarms within hypoglycemic events as described in [19], but considered 
only the first alarm within 60 min of each hypoglycemic event.

We calculate the false alarm rate metric, which represents the false alarm ratio among the number of times a patient is alarmed of a hypoglycemic event, as follows:

$$
\text { False Alarm Rate }(\mathrm{FAR})=\frac{F P_{e}}{T P_{e}+F P_{e}}
$$

where $T P_{e}$ is true positive for an event and $F P_{e}$ is the false positive for an event.

\section{Results}

We analyzed 107 three-day CGM datasets from 104 patients (type- $1, n=52$; type- $2, n=52$ ). In this 107 three-day CGM datasets (99,955 CGM data points at 5-min intervals, the average number of CGM data points per CGM time series was 934), $10.4 \%$ had a glucose level $<3.9 \mathrm{mmol} / \mathrm{L}$ (70 $\mathrm{mg} / \mathrm{dL})$. After preprocessing of data, 2062 samples of

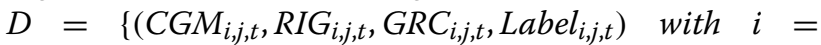
$1, \ldots, 107, j=1, \ldots, M_{i}$, and $\left.t=1, \ldots, 42\right\}$ were included with Lable $_{i, j, t}=1$ and remaining were samples with Lable $_{i, j, t}=0$. The labels with Lable $_{i, j, t}=0$ amounts to about 16.1 times the total number of samples with Lable $_{i, j, t}=1$. This difference represents a high data imbalance between the numbers of occurrence of hypoglycemia vs nonoccurrence of hypoglycemia after mealtimes. The total number of postprandial hypoglycemic events within $5 \mathrm{~min}$ to $4 \mathrm{~h}$ after each meal announcement was 249 .

After we trained machine learning models (RF, SVMLN, SVM-RBF, KNN, and LR) with train set $q$ obtained from $q^{\text {th }}$ training folds, we calculated the statistical performance of each model on each individual test set $q$. Since the 5-fold cross-subject validation was done, 5 performances of each model were used to determine the average performance. The averaged results are summarized in Table 2.

In order to identify the difference between the average metrics of multiple models, we used statistical analysis methods. The first way is to find models showed the highest metric or the lowest metric, and the second way is to use a one-way analysis of variance (ANOVA) or the Kruskal-Wallis Rank Sum Test for finding a significant difference on the average of a metric of the models. We used the one-way ANOVA only when both normality with the Shapiro-Wilk test and homoscedasticity with Bartlett's test were satisfied. Otherwise, the Kruskal-Wallis Rank Sum Test was used.

As shown in Table 2, the average AUC's of RF, SVM-LN, SVM-RBF, KNN, and LR were 0.966, 0.967, 0.952, 0.917 and 0.967 , respectively. All five machine learning models showed high AUC, with LR showing slightly better performance compared to others, implying that the machine learning models have high accuracy and excellent predictive ability [39]. When we used the one-way ANOVA on AUC, there is a significant difference $(p<0.05)$. It indicates that KNN shows the worst AUC. Figure 4 shows ROC curves of the different models.

In sensitivity, LR showed the highest average sensitivity (93.6\%) and KNN showed the lowest average sensitivity (88.5\%). When we used the Kruskal-Wallis Rank Sum Test on sensitivity, there is no significant difference ( $p$ $=0.06$ ), but the $\mathrm{p}$-value is very close to 0.05 . In specificity, RF showed the highest average specificity (91.3\%) and LR showed the lowest average specificity (87.9\%). In F1 score, RF showed the highest average F1 score (0.543) and LR showed the lowest average F1 score (0.484). In $T P_{e}$, RF showed the highest average value (30.2) and SVMLN showed the lowest average value (29.2). In FAR, KNN showed the highest average FAR (0.779) and RF showed the lowest average FAR (0.704). In DT, KNN showed the highest DT (25.8) and LR showed the lowest DT (25.0). With the one-way ANOVA on the metrics including sensitivity, specificity, F1 score, $T P_{e}$, and DT, there is no significant difference $(p>0.05)$. On the other hand, with the one-way ANOVA on FAR, there is a significant difference $(p=0.046<0.05)$. This indicates that RF is able to be a good model to predict postprandial hypoglycemia.

Since clinically severe hypoglycemia (glucose level less than $3.0 \mathrm{mmol} / \mathrm{L}$, i.e., that is about $54 \mathrm{mg} / \mathrm{dL}$ [34]) can lead to catastrophic health issues [35], any predictive model must accurately alarm this clinically significant hypoglycemic events. To calculate the performance of clinically-severe-hypoglycemia alarms, the number of alarmed clinically significant hypoglycemic events was counted for each model. For example, if the alarmed hypoglycemic event has at least one CGM data point $<3.0$ $\mathrm{mmol} / \mathrm{L}$, we regard this event as an alarmed clinically significant hypoglycemic event. On the other hand, if a missed hypoglycemic event has at least one CGM data point $<3.0 \mathrm{mmol} / \mathrm{L}$, we consider this event as a missed clinically significant hypoglycemic event. With the 5 -fold cross-subject validation, the average number of the hypoglycemic events including at least one CGM data point $<3.0 \mathrm{mmol} / \mathrm{L}$ was 16.6 . The average number of alarmed clinically significant hypoglycemic events made by RF was 14.4 events, by SVM-LN was 14.6 events, by SVMRBF was 14.2 events, by KNN was 15 events, and LR was 14.4 events. All models alarmed clinically significant hypoglycemic events of more than $86 \%$. With the one-way ANOVA on the alarmed clinically significant hypoglycemic events, there is no significant difference ( $p$ $=0.989$ ).

To reveal that a part of false alarms were useful, we considered near-hypoglycemic events that includes at least two consecutive CGM data points $\leq 4.4 \mathrm{mmol} / \mathrm{L}$, i.e., about $80 \mathrm{mg} / \mathrm{dL}$ [40]. In other words, we counted all false 
Table 2 Average and standard deviation of metrics of models with 5-fold cross-subject validation

\begin{tabular}{|c|c|c|c|c|c|c|c|c|}
\hline Model & Sen $(\%, S D)$ & Spe $(\%, S D)$ & F1 score (SD) & $A \cup C(S D)$ & $\mathrm{NH}(\mathrm{SD})$ & $T P_{e}(\mathrm{SD})$ & FAR (SD) & $\mathrm{DT}(\min , \mathrm{SD})$ \\
\hline \multirow{2}{*}{ RF } & 89.6 & 91.3 & 0.543 & 0.966 & 36.4 & 30.2 & 0.704 & 25.5 \\
\hline & $(2.78)$ & $(2.03)$ & $(0.053)$ & $(0.007)$ & $(11.0)$ & $(8.42)$ & $(0.035)$ & $(1.97)$ \\
\hline SVM & 93.3 & 88.2 & 0.487 & 0.967 & 36.4 & 29.2 & 0.777 & 25.8 \\
\hline$-L N$ & $(1.70)$ & $(2.83)$ & $(0.046)$ & $(0.007)$ & $(11.0)$ & $(8.30)$ & $(0.034)$ & $(2.12)$ \\
\hline SVM & 89.9 & 88.8 & 0.487 & 0.952 & 36.4 & 29.4 & 0.760 & 25.2 \\
\hline$-\mathrm{RBF}$ & $(8.65)$ & $(2.96)$ & $(0.062)$ & $(0.014)$ & $(11.0)$ & $(9.20)$ & $(0.038)$ & $(3.22)$ \\
\hline \multirow{2}{*}{ KNN } & 88.5 & 89.4 & 0.492 & 0.917 & 36.4 & 29.6 & 0.779 & 25.8 \\
\hline & $(1.93)$ & $(2.09)$ & $(0.054)$ & $(0.012)$ & $(11.0)$ & $(8.73)$ & $(0.038)$ & $(3.76)$ \\
\hline \multirow{2}{*}{ LR } & 93.6 & 87.9 & 0.484 & 0.967 & 36.4 & 29.6 & 0.772 & 25.0 \\
\hline & $(2.25)$ & $(2.95)$ & $(0.047)$ & $(0.007)$ & $(11.0)$ & $(8.71)$ & $(0.037)$ & $(2.87)$ \\
\hline
\end{tabular}

With the 5-fold cross-subject validation, average metrics were calculated using Eq 6, 7, 9, and 10 on test set $q, q=1,2,3,4,5$. Since there should be at least two consecutive predictions of a hypoglycemia alert value to make an alarm, we excluded hypoglycemic events occurring immediately after meals. Abbreviation: RF, random forest; SVM-LN, support vector machine with a linear kernel; SVM-RBF, support vector machine with a radial basis function; KNN, K-nearest neighbor; LR, logistic regression; SD, standard deviation; Sen, sensitivity; Spe, specificity; AUC, the area under the ROC curve; NH, the number of hypoglycemic events; FAR, false alarm rate; DT, detection time.

alarms where near-hypoglycemic events were included within $60 \mathrm{~min}$ after the alarms. The $40.0 \%$ of average false alarms of RF were related to the near-hypoglycemic events, $28.6 \%$ of average false alarms of SVM-LN were related to the near-hypoglycemic events, $31.3 \%$ of average false alarms of SVM-RBF were related to the events, $29.9 \%$ of average false alarms of KNN were related to the events, and $29.1 \%$ of average false alarms of LR were related to the events. With the one-way ANOVA on the percentage, there is a significant difference $(p<0.05)$. This result indicates many of false alarms made by the models were related to the near-hypoglycemic events and also indicates that the average false alarms of the RF was not only lowest, but also many RF's false alarms were associated with the near-hypoglycemic events.

As a result, RF is better in predicting postprandial hypoglycemia with the high level of predictability.

We are the first to use RIG as a new feature. To validate the impact of the feature, we trained another RF considering only two features (i.e., CGM and GRC). As a result, the RF showed 92.2 (4.11) \% of the average sensitivity, 89.1 (3.34) \% of the average specificity, 0.509 (0.051) of the average F1 score, 0.961 (0.007) of the average AUC, 29.4 (9.39) of the average $T P_{e}, 0.742(0.038)$ of the average FAR, and $25.7(2.48) \mathrm{min}$ of the average DT. When we compared the RF trained by all features with the RF trained by two features (i.e., CGM and GRC), there are significant differences in AUC $(p=0.033<0.05)$ and FAR $(p=0.045<0.05)$ with Paired t-test. These results establish the importance of RIG in improving the performance.

\section{Discussion}

In this study, the contributions are three folds. First, we verified the feasibility of the RF-based classifier with the simple feature set for predicting postprandial hypoglycemia. In comparing with other commonly used machine learning models, the RF showed the best predictive capabilities with the highest average AUC and superior statistical performance. Second, the proposed methodology uses only a few CGM data points and simple meal announcements. It does not require patients to manually calculate and enter the complex information such as carbohydrate intakes and insulin information. This will minimize patients' burdens and eventually lower the risk of mistaking data inputs. Third, we found a unique datadriven feature set by intensive review of patient glucose data. The feature set includes the useful RIG (the rate of increase of glucose after a meal) which reflects the steep increase in a glucose level after a meal because of intake of foods with high glycemic index or the late timing of premeal rapid-acting insulin. Moreover, the presence of a postprandial meal peak glucose due to a small amount of meal and a low peak is reflected in RIG. In addition, our study was based on quite large dataset from patients with both types of diabetes (107 CGM cases from 104 patients including 52 people with type 1 diabetes and 52 people with type 2 diabetes), and thus we expect that our proposed method can fit to a general case to predict and prevent postprandial hypoglycemia.

Training models with highly imbalanced dataset is a technically challenging task. This can cause a serious performance distortion. As mentioned in the "Results" section, there was a high imbalance (16.1 times) between hypoglycemia and non-hypoglycemia. This data imbalance problem can be solved using approaches [41] such as over/under sampling, cost-based learning, etc. Among these approaches, we used the cost-sensitive learning to utilize full data samples and avoid training with redundant data samples. We have assigned different costs between FP and TN to solve the problem of highly imbalanced dataset, and have trained the models to predict 


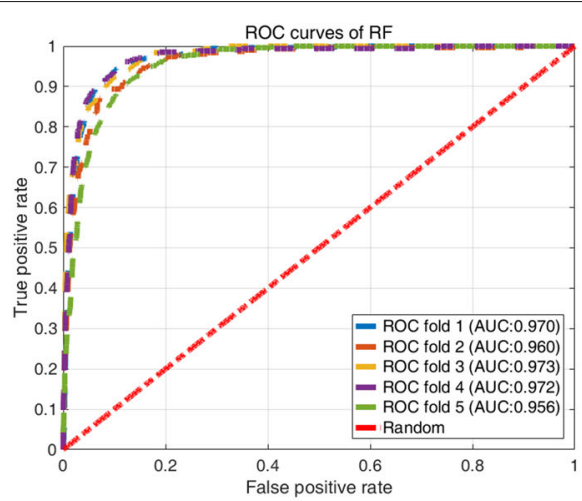

(a)

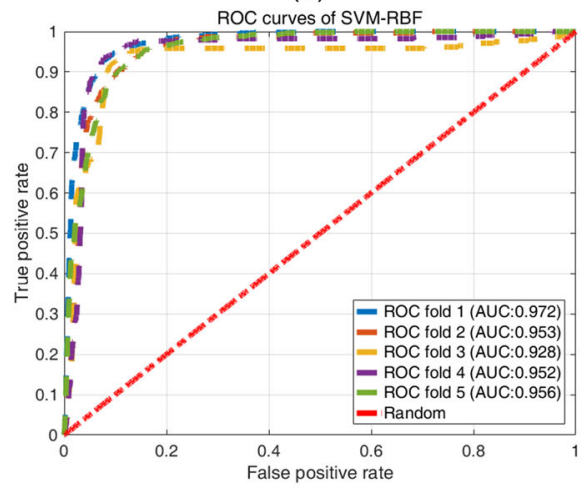

(c)

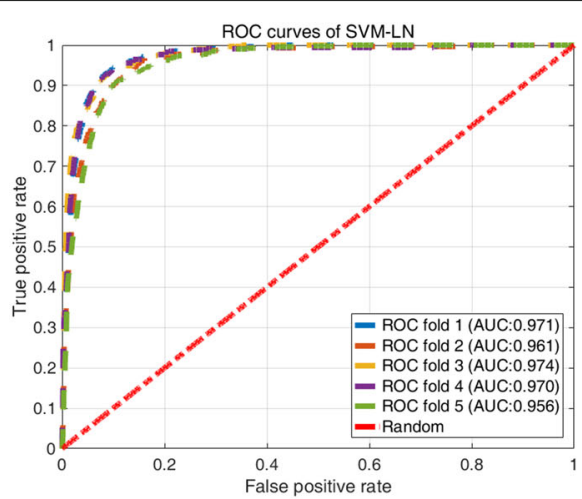

(b)

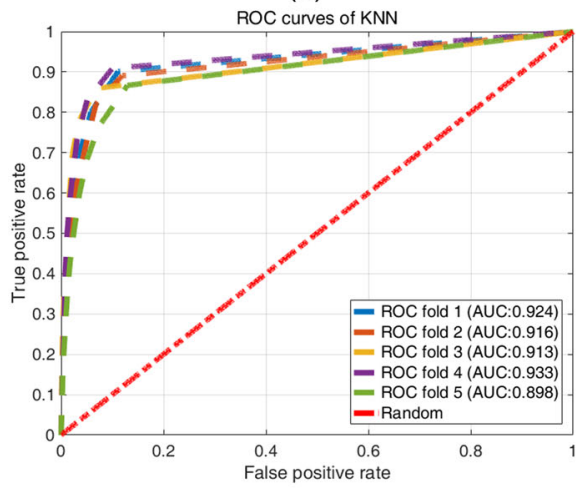

(d)

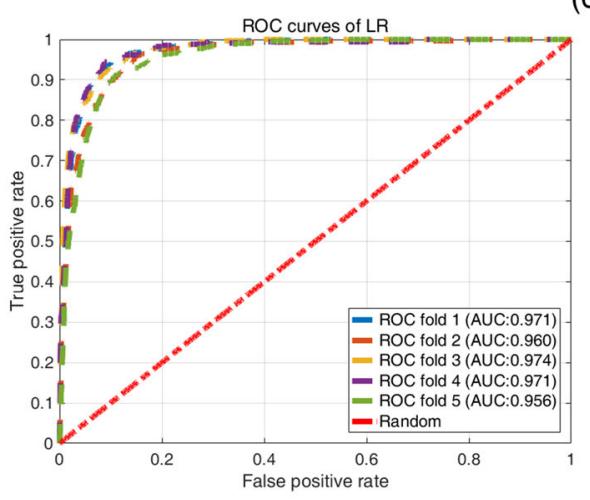

(e)

Fig. 4 ROC curves for different models. In each iteration of the 5-fold cross-subject validation, the hyper-parameters of the models were determined by the grid search method. a ROC curves of RF. b ROC curves of SVM-LN. c ROC curves of SVM-RBF. d ROC curves of KNN. e ROC curves of LR. Each colored dashed line represents the ROC curve of each fold. The red dash-dot line indicates a random prediction (i.e., $A \cup C=0.5$ )

more hypoglycemia. As a result, the five models showed the high sensitivity greater than $88 \%$, big $T P_{e}$, and the large number of alarmed events including at least one CGM data point $<3.0 \mathrm{mmol} / \mathrm{L}$ (about $54 \mathrm{mg} / \mathrm{dL}$ ), which is considered as clinically significant hypoglycemia. Although these results have showed high FAR, many false alarms have been found to be associated with near-hypoglycemic events that have two consecutive CGM data points $\leq 4.4$ $\mathrm{mmol} / \mathrm{L}$ (about $80 \mathrm{mg} / \mathrm{dL}$ ). This means that there is a lot of glucose fluctuations around mealtimes. Unlike nocturnal hypoglycemia, it is because there may be unpredictable interventions from people with diabetes in the daytime. For example, the patients may be exercising, stressed, or taking sugary drinks or snacks. Accurate predictions of postprandial may require more user unfriendly manual inputs, but this increases the burden on the patient and the chance of users' mistakes in entering information. Thus, it is necessary to develop a system that can automatically process the patient's lifestyle data, or more studies, which predict the occurrence of postprandial hypoglycemia with only CGM measurements and easy input, are needed. 
Our results showed that the ensemble way that uses multiple single learners to make a decision with a voting has the better predictability than the single model such as SVM-LN, SVM-RBF, KNN, and LR. This implies that the ensemble approach has better generalization capabilities compared to other models on predictions of the occurrence of postprandial hypoglycemia during various glycemic changes which are affected by carbohydrate in a meal and injected insulin doses. Thus, we selected RF as our primary model to predict the occurrence of postprandial hypoglycemia, and other model were used to contrast the performance. For future advanced studies, the process that optimizes the structure of an ensemble method such as stacking multiple models, soft voting, and hard voting and selects appropriate machine learning models is needed. Furthermore, it is also necessary to take into account the computational complexity of the ensemble model for working on a compact device. Although this process may require complex procedures and lots of time, it is expected that it will enable the development of a model predicting more accurately the occurrence of postprandial hypoglycemia without any manual inputs.

For patients, the extended prediction horizon is beneficial because it increases the time available for a patient to take action to prevent potential hypoglycemia. However, it should be noted that there is a trade-off relationship between the prediction horizon and the accuracy of a model [42]. Generally, increasing the prediction horizon will lower the accuracy and priority should be decided based on clinical needs. For example, patients who want to know the occurrence of hypoglycemia earlier, in spite of many false alarms, will prefer a long-term prediction horizon. Conversely, a short-term prediction horizon will be preferred for patients who want to know the occurrence of hypoglycemia with higher confidence. The primary reason for choosing the 30-min prediction horizon was the good trade-off between the prediction horizon and the accuracy of prediction [37]. The 30-min prediction horizon enabled an acceptable accuracy while providing an effective time for correcting hypoglycemia with carbohydrate ingestion or injection of glucagon. In addition, several studies have used the 30 -min prediction horizon $[17-21,23,37,43]$ and have verified that this time is sufficient to prevent hypoglycemia in patients [17]. Therefore, we believe the 30-min prediction horizon used in this study would be adequate for alarming people with type 1 diabetes to take carbohydrate or for alarming a bihormonal AP system to infuse glucagon, but the 30-min prediction horizon might be inadequate for prevention of hypoglycemia only by reduction of insulin infusion rate in single hormone AP system.

Besides AP system, the model is also useful for standalone real-time CGM device since it requires only mealtime announcement and CGM data for its operation. Meal announcement is manual but can easily performed by pressing a button on the device. In many type-1 or insulintreated type- 2 diabetes patients, who cannot use an insulin pump, multiple daily injection insulin therapy with real-time CGM is a reasonable option [44]. Widespread use of flash BG monitoring, which can replace a fingerstick glucometer even for insulin-treated type-2 diabetes patients. In these clinical settings, the feature of our algorithm that does not require insulin dosing information could be a benefit for patients who do not use insulin pumps [45].

To collect three-day CGM data points from 104 people with type 1 and type 2 diabetes, Medtronic's CGMS Gold $^{T M}$ was used. This device retrospectively calibrated and filtered collected CGM data points at the end of the monitoring. Thus, the collected CGM traces are smoother than the real-time CGM traces.

It is important to acknowledge the limitations of the study. First, tests were performed retrospectively with 107 three-day CGM datasets, and a prospective study should be conducted to assess the clinical credibility of the prediction algorithm. Second, 30-minute, which was the prediction horizon, may be not an enough time to avoid hypoglycemia without the ingestion of carbohydrates or injection of glucagon. Last, a patient should announce mealtimes to operate our algorithm. Although it adds a manual activity to the patient, it is still a much less burdensome activity than counting carbohydrates and entering injected insulin dose that other algorithms require. As the next step, we will develop a meal detection algorithm by using CGM data and accumulated patients' mealtime information. It is expected to greatly improve the usability of the hypoglycemia prediction algorithm.

\section{Conclusions}

In this study, we could successfully identify hypoglycemia using the RF-based model in the postprandial situation. The algorithm could predict a hypoglycemia alert value in a clinically-useful 30-min prediction horizon around mealtimes. This proposed approach only requires CGM data points and simple mealtime announcements, and is less burdensome to patients than models using lots of input information. This study not only establishes a new methodology to predict postprandial hypoglycemia but also verifies the feasibility of RF to accurately predict postprandial hypoglycemia. We believe that the proposed machine learning approach can be integrated with realtime CGM devices and sensor-based AP system, so it will be a great help for people with diabetes to manage their glucose level and improve their quality of life. In the near future, we will evaluate our algorithm on a prospective patient population to clearly establish the clinical use of this system. 


\section{Abbreviations}

AP: Artificial pancreas; CGM: Continuous glucose monitoring; CSII: Continuous subcutaneous insulin infusion; FAR: False alarm rate; FGM: Flash glucose monitoring; KNN: K-nearest neighbor; MDI: Multiple daily injection; RF: Random forest; SVM-LN: Support vector machine with a linear kernel; SVM-RBF: Support vector machine with a radial basis function

\section{Acknowledgements}

We are grateful to Min Ja Cho (Diabetes Center, Division of Endocrinology and Metabolism, Department of Medicine, Samsung Medical Center) for her dedication to the CGMS data collection and management.

\section{Authors' contributions}

WS was a major contributor in writing the manuscript. WS reviewed related works and designed the study. Also, he conducted main data mining and all post-hoc analysis. YBL conducted data collection, and analysed and summarized the clinical aspects of study subjects. SL conducted data preprocessing. SMJ and YBL contributed on manuscript writing and critical revision of the background, discussion and conclusion parts. SMJ and SMP managed the whole process, and contributed on the final approval of manuscript. All authors read and approved the final manuscript.

\section{Funding}

This research was supported by the Ministry of Science and ICT (MSIT), Korea, under the ICT Consilience Creative program (IITP-2019-2011-1-00783) supervised by the Institute for Information and communications Technology Promotion (IITP), which provide financial support in the anlaysis and interpretation of data, and the Basic Science Research Program through the National Research Foundation of Korea (NRF) funded by the Ministry of Science and ICT (NRF-2017R1A5A1015596), which provide financial support in the anlaysis and interpretation of data. The publication of this article and writing the manuscript were funded by the Technology Innovation Program or Industrial Strategic Technology Development Program (20001841, Development of System for Intelligent ContextAwareWearable Service based on Machine Learning) funded By the Ministry of Trade, Industry and Energy (MOTIE, Korea). The funding sources had no role in the design of the study and data collection.

\section{Availability of data and materials}

The data that support the findings of this study are available from Samsung Medical Center but restrictions apply to the availability of these data. The data were used under license for the current study, and so are not publicly available. Data are however available from the authors upon reasonable request and with permission of Samsung Medical Center.

\section{Ethics approval and consent to participate}

The protocol of this study was approved by the Institutional Review Board (IRB) of the Samsung Medical Center. The informed consent requirement for this study was waived because the researcher only accessed the database for analysis purposes, and all patient data were de-identified (IRB file number; SMC 2016-05-058-001) All medical procedures were performed in accordance with relevant guidelines and regulations. The informed consent requirement for this study was waived by the board because the researcher only accessed the database for analysis purposes, and all patient data were de-identified.

\section{Consent for publication}

Not applicable.

\section{Competing interests}

The authors declare that they have no competing interests.

Received: 12 February 2019 Accepted: 21 October 2019 Published online: 06 November 2019

\section{References}

1. Nathan DM, DCCT/Edic Research Group. The diabetes control and complications trial/epidemiology of diabetes interventions and complications study at 30 years: overview. Diabetes Care. 2014;37:9-16.

2. Phillip M, Battelino T, Atlas E, Kordonouri O, Bratina N, Miller $\mathrm{S}$, et al. Nocturnal glucose control with an artificial pancreas at a diabetes camp. N Engl J Med. 2013;368:824-33.
3. Thabit H, Tauschmann M, Allen J, Leelarathna L, Hartnell S, Wilinska M, et al. Home Use of an Artificial Beta Cell in Type 1 Diabetes. New Engl $J$ Med. 2015;373:2129-40.

4. Diabetes Control and Complications Trial/Epidemiology of Diabetes Interventions and Complications (DCCT/EDIC) Study Research Group. Intensive diabetes treatment and cardiovascular disease in patients with type 1 diabetes. N Engl J Med. 2005;353:2643-53.

5. Cryer PE. The barrier of hypoglycemia in diabetes. Diabetes. 2008;57: 3169-76.

6. Russell SJ, El-Khatib FH, Sinha M, Magyar KL, McKeon K, Goergen LG, et al. Outpatient Glycemic Control with a Bionic Pancreas in Type 1 Diabetes. N Engl J Med. 2014;371:313-25.

7. Kudva YC, Carter RE, Cobelli C, Basu R, Basu A. Closed-loop artificial pancreas systems: Physiological input to enhance next-generation devices. Diabetes Care. 2014;37:1184-90.

8. Kovatchev B, Tamborlane WV, Cefalu WT, Cobelli C. The artificial pancreas in 2016: A digital treatment ecosystem for diabetes. Diabetes Care. 2016;39(7):1123-26. https://doi.org/10.2337/dc16-0824.

9. Bergenstal RM, Garg S, Weinzimer SA, Buckingham BA, Bode BW, Tamborlane WW, et al. Safety of a Hybrid Closed-Loop Insulin Delivery System in Patients With Type 1 Diabetes. JAMA. 2016;316:1407.

10. Rylander D. Glucagon in the Artificial Pancreas: Supply and Marketing Challenges. J Diabetes Sci Technol. 2015;9(1):52-55. https://doi.org/10. 1177/1932296814546668.

11. Pohl R, Li M, Krasner A, De Souza E. Development of stable liquid glucagon formulations for use in artificial pancreas. J Diabetes Sci Technol. 2015;8(1):8-16.

12. Haidar A, Rabasa-Lhoret R, Legault L, Lovblom LE, Rakheja R, Messier V, D'Aoust É, Falappa CM, Justice T, Orszag A. Single- and dual-hormone artificial pancreas for overnight glucose control in type 1 diabetes. J Clin Endocrinol Metab. 2016;101(1):214-23.

13. Home PD. Plasma insulin profiles after subcutaneous injection: How close can we get to physiology in people with diabetes? Diabetes Obes Metab. 2015;17(11):1011-20. https://doi.org/10.1111/dom.12501.

14. Unger RH, Orci L. Paracrinology of islets and the paracrinopathy of diabetes. Proc Natl Acad Sci. 2010;107:16009-12.

15. Oviedo S, Vehi J, Calm R, Armengol J. A review of personalized blood glucose prediction strategies for T1DM patients. Int I Numer Method Biomed Eng. 2017;33(6):1-21.

16. Woldaregay AZ, Årsand E, Botsis T, Albers D, Mamykina L, Hartvigsen G. Data-Driven Blood Glucose Pattern Classification and Anomalies Detection: Machine-Learning Applications in Type 1 Diabetes. J Med Internet Res. 21(5):e11030.

17. Sparacino G, Zanderigo F, Corazza S, Maran A, Facchinetti A, Cobelli C. Glucose concentration can be predicted ahead in time from continuous glucose monitoring sensor time-series. IEEE Trans Biomed Eng. 2007;54: 931-7.

18. Yang J, Li L, Shi Y, Xie X. An ARIMA Model with Adaptive Orders for Predicting Blood Glucose Concentrations and Hypoglycemia. IEEE J Biomed Health Inform. 2018;23(3):1251-60.

19. Turksoy K, Bayrak ES, Quinn L, Littlejohn E, Rollins D, Cinar A. Hypoglycemia early alarm systems based on multivariable models. Ind Eng Chem Res. 2013;52:12329-36.

20. Pérez-Gandía C, Facchinetti A, Sparacino G, Cobelli C, Gómez EJ, Rigla M, et al. Artificial neural network algorithm for online glucose prediction from continuous glucose monitoring. Diabetes Technol Ther. 2010;12:81-8.

21. Zecchin C, Facchinetti A, Sparacino G, De Nicolao G, Cobelli C. Neural network incorporating meal information improves accuracy of short-time prediction of glucose concentration. IEEE Trans Biomed Eng. 2012;59: 1550-60.

22. Zecchin C, Facchinetti A, Sparacino G, De Nicolao G, Cobelli C. A new neural network approach for short-term glucose prediction using continuous glucose monitoring time-series and meal information. In: Proceedings of the Annual International Conference of the IEEE Engineering in Medicine and Biology Society, EMBS; 2011. p. 5653-6. https://doi.org/10.1109/iembs.2011.6091368.

23. Zecchin C, Facchinetti A, Sparacino G, Cobelli C. How much is short-term glucose prediction in type 1 diabetes improved by adding insulin delivery and meal content information to CGM data? A proof-of-concept study. J Diabetes Sci Technol. 2016;10:1149-60. 
24. Pappada SM, Cameron BD, Rosman PM, Bourey RE, Papadimos TJ, et al. Neural network-based real-time prediction of glucose in patients with insulin-dependent diabetes. Diabetes Technol Ther. 2011;13:135-41.

25. Dassau E, Cameron F, Lee H, Bequette BW, Zisser H, Jovanovič L, et al. Real-Time Hypoglycemia Prediction Suite Using Continuous Glucose Monitoring. Diabetes Care. 2010;33:1249-54.

26. Eljil KS, Qadah G, Pasquier M. Predicting hypoglycemia in diabetic patients using data mining techniques. 2013 9th Int Conf Innov Inf Technol. 2013130-5. https://doi.org/10.1109/innovations.2013.6544406.

27. Miyeon J, et al. Prediction of Daytime Hypoglycemic Events Using Continuous Glucose Monitoring Data and Classification Technique. arXiv preprint. 2017. https://arxiv.org/abs/1704.08769.

28. Sudharsan B, Peeples M, Shomali M. Hypoglycemia prediction using machine learning models for patients with type 2 diabetes. J Diabetes Sci Technol. 2015;9(1):86-90. https://doi.org/10.1177/1932296814554260.

29. Liebl A, Henrichs HR, Heinemann L, Freckmann G, Biermann E, Thomas A. Continuous glucose monitoring: Evidence and consensus statement for clinical use. J Diabetes Sci Technol. 2013;7:500-19.

30. The Diabetes Research in Children Network (DirectNet) Study Group. The accuracy of the CGMSTM in children with type 1 diabetes: results of the Diabetes Research in Children Network (DirecNet) accuracy study. Diabetes Technol Ther. 2003;5(5):781-789. https://doi.org/10.1089/ 152091503322526987.

31. Zhong WW, Crandell JL, Shay CM, Gordon-Larsen P, Cole SR, Juhaeri J, et al. Dietary intake and risk of non-severe hypoglycemia in adolescents with type 1 diabetes. J Diabetes Complicat. 2017;31(8):1340-7. https://doi.org/ 10.1016/j.jdiacomp.2017.04.017.

32. Joubert M, Baillot-Rudoni S, Catargi B, Charpentier G, Esvant A, Franc S, et al. Indication, organization, practical implementation and interpretation guidelines for retrospective CGM recording: A French position statement. Diabetes Metab. 2015;41(6):498-508. https://doi.org/ 10.1016/j.diabet.2015.07.001.

33. Palerm CC, Willis JP, Desemone J, Bequette BW. Hypoglycemia Prediction and Detection Using Optimal Estimation. Diabetes Technol Ther. 2005;7:3-14.

34. American Diabetes Association. 6. Glycemic targets: standards of medical care in diabetes-2018. Diabetes Care. 2018;41(Supplement 1):S55-64. https://doi.org/10.2337/dc18-s006.

35. International Hypoglycaemia Study Group. Glucose concentrations of less than $3.0 \mathrm{mmol} / \mathrm{L}(54 \mathrm{mg} / \mathrm{dL}$ ) should be reported in clinical trials: a joint position statement of the American Diabetes Association and the European Association for the Study of Diabetes. Diabetes Care. 2017;40: 155-7.

36. Schaller HC, Schaupp L, Bodenlenz M, Wilinska ME, Chassin L, Wach P, et al. On-line adaptive algorithm with glucose prediction capacity for subcutaneous closed loop control of glucose: Evaluation under fasting conditions in patients with Type 1 diabetes. Diabet Med. 2006;23(1):90-3. https://doi.org/10.1111/j.1464-5491.2006.01695.x.

37. Gadaleta M, Facchinetti A, Grisan E, Rossi M. Prediction of Adverse Glycemic Events from Continuous Glucose Monitoring Signal. IEEE $J$ Biomed Health Inform. 2018;23(2):650-9. https://doi.org/10.1109/jbhi. 2018.2823763.

38. Ganganwar V. An overview of classification algorithms for imbalanced datasets. J Emerg Technol Adv Eng. 2012;2:42-7.

39. Akobeng AK. Understanding diagnostic tests 3 : Receiver operating characteristic curves. Acta Paediatr Int J Paediatr. 2007;9(3):145. https:// doi.org/10.4103/picr.picr_87_18.

40. Plis K, Bunescu R, Marling C, Shubrook J, Schwartz F. A Machine Learning Approach to Predicting Blood Glucose Levels for Diabetes Management. In: Workshops at the Twenty-Eighth AAAI Conference on Artific ial Intelligence: 2014; 2014.

41. Longadge R, Dongre S, Malik L. Class imbalance problem in data mining review. 2013. arXiv preprint arXiv:13051707.

42. Palerm CC, Willis JP, Desemone J, Bequette BW. Hypoglycemia detection and prediction using continuous glucose monitoring - A study on hypoglycemic clamp data. J Diabetes Sci Technol. 2007;5(1):624-9. https://doi.org/10.1177/193229680700100505.

43. Mhaskar HN, Pereverzyev SV, van der Walt MD. A deep learning approach to diabetic blood glucose prediction. Front Appl Math Stat. 2017;3:14. https://doi.org/10.3389/fams.2017.00014.
44. Perlmuter LC, Flanagan BP, Shah PH, Singh SP. Glycemic Control and Hypoglycemia: Is the loser the winner? Diabetes Care. 2008;31:2072-6.

45. Heinemann L, Freckmann G. CGM versus FGM; or, continuous glucose monitoring is not flash glucose monitoring. J Diabetes Sci Technol. 2015;9(5):947-50. https://doi.org/10.1177/1932296815603528.

\section{Publisher's Note}

Springer Nature remains neutral with regard to jurisdictional claims in published maps and institutional affiliations.
Ready to submit your research? Choose BMC and benefit from:

- fast, convenient online submission

- thorough peer review by experienced researchers in your field

- rapid publication on acceptance

- support for research data, including large and complex data types

- gold Open Access which fosters wider collaboration and increased citations

- maximum visibility for your research: over $100 \mathrm{M}$ website views per year

At BMC, research is always in progress.

Learn more biomedcentral.com/submissions 